\title{
FLUJOS DE ÓXIDO NITROSO EN PASTURAS BAJO DIFERENTES DOSIS DE FERTILIZACIÓN NITROGENADA, NORTE DE COLOMBIA*
}

\author{
'José Luis Contreras-Santos ${ }^{1 / * *}$, Manuel Ramón Espinosa-Carvajal ${ }^{2}$, Jorge Cadena-Torres $^{3}$, \\ Judith Martínez-Atencia ${ }^{4}$, Cindy Katherine Falla-Guzmán ${ }^{5}$, Jeyson Fernando Garrido-Pineda ${ }^{6}$ \\ Palabras clave: Gases de efecto invernadero (GEI); mitigación; gramínea; \\ propiedades físico-químicas suelo; producción ganadera. \\ Keywords: Greenhouse gas; mitigation; grass; physical-chemical properties of soil; \\ livestock-bovine.
}

Recibido: 28/07/2021

RESUMEN

Introducción. El óxido nitroso es un gas de efecto invernadero de gran impacto sobre el calentamiento global. En los sistemas de producción ganaderos es de gran importancia buscar alternativas que permitan mitigar las emisiones de $\mathrm{N}_{2} \mathrm{O}$, dentro de las cuales se ha considerado el uso de coberturas vegetales capaces de reducir el proceso de nitrificación. Objetivo. Evaluar la dinámica de los flujos de $\mathrm{N}_{2} \mathrm{O}$ en suelos bajo ocupación ganadera cubiertos con gramíneas mejoradas y fertilización nitrogenada (Úrea). Materiales y métodos. Esta investigación se desarrolló en un sistema de producción de carne en el valle medio del Rio Sinú, en el cual los flujos de $\mathrm{N}_{2} \mathrm{O}$ fueron monitoreados durante un

* Resultados derivados de proyecto de investigación "Producción intensiva de carne en pastos con diferente capacidad para inhibir la nitrificación y reducir las emisiones de gases de efecto invernadero", financiado por el Ministerio de Agricultura y Desarrollo Rural (MADR) y la Corporación Colombiana de Investigación Agropecuaria (Agrosavia).

** Autor para correspondencia. Correo electrónico: jlcontreras@agrosavia.co

1 Corporación Colombiana de Investigación Agropecuaria (AGROSAVIA), C.I Turipaná, Colombia.

(D) 0000-0002-8179-3430. Agropecuaria (AGROSAVIA), C.I Turipaná, Colombia Sede Carmen De Bolívar.

(D) 0000-0001-8939-5472.

Aceptado: 24/08/2021

\section{ABSTRACT}

Nitrous oxide fluxes in pastures under different dose of nitrogen fertilization, Northern Colombia. Introduction. Nitrous oxide is a highly relevant greenhouse gas. In livestock production systems, it is of a great importance to search for alternatives to mitigate $\mathrm{N}_{2} \mathrm{O}$ emissions, among which the use of plant covers capable of reducing nitrification process has been considered. Objective. Evaluate the dynamics of $\mathrm{N}_{2} \mathrm{O}$ fluxes in livestock soils covered with two improved grasses and doses of nitrogen fertilization (Úrea). Materials and methods. This research was developed in a livestock system in the middle valley of the Rio Sinú, in which $\mathrm{N}_{2} \mathrm{O}$ fluxes were monitored during

\footnotetext{
3 Corporación Colombiana De Investigación Agropecuaria (AGROSAVIA), C.I Turipaná, Colombia (D) 0000-0002-5180-2893.

4 Corporación Colombiana De Investigación Agropecuaria (AGROSAVIA), C.I Turipaná, Colombia. (iD) 0000-0002-8275-2956.

5 Corporación Colombiana De Investigación Agropecuaria (AGROSAVIA), C.I Turipaná, Colombia. (D) 0000-0002-1266-8505.

6 Corporación Colombiana De Investigación Agropecuaria (AGROSAVIA), C.I Turipaná, Colombia. (D) 0000-0002-1405-1066.
} 
periodo de 12 meses (noviembre 2014 - 2015). Se utilizó un diseño experimental de bloques completos al azar, en arreglo de parcelas divididas y 2 repeticiones, en donde la parcela principal correspondió a las gramíneas (Brachiaria humidicola CIAT679 y Panicum maximum cv. Tanzania) y suelo descubierto, y las subparcelas a 3 dosis de fertilización nitrogenada $(0,150,300$ kg.ha $\left.{ }^{-1} \cdot a_{n} o^{-1} N\right)$. Resultados. Los flujos de $\mathrm{N}_{2} \mathrm{O}$ fluctuaron según el contenido de humedad del suelo, asociado a los periodos de mayor o menor precipitación en la región. La cobertura con la gramínea Brachiaria humidicola cv CIAT679 mostró menores emisiones de $\mathrm{N}_{2} \mathrm{O}$, mientras que las dosis de fertilizantes nitrogenados aumentaron las emisiones de $\mathrm{N}_{2} \mathrm{O}$. Dichos efectos fueron en parte atenuados por el uso de la cobertura con Brachiaria humidicola CIAT679. Conclusiones. Los resultados obtenidos indican que las emisiones de óxido nitroso en los sistemas ganaderos podrían variar a partir del tipo de cobertura y las dosis de fertilización nitrogenada utilizadas. En este caso, se encontró que la cobertura con Brachiaria humidicola cv CIAT679 en los sistemas de producción ganaderos reduce en más del $40 \%$ las emisiones de óxido nitroso hasta una dosis de $\mathrm{N}$ máxima de $300 \mathrm{~kg} \cdot \mathrm{ha}^{-1} \cdot$ año $^{-1}$.

\section{INTRODUCCIÓN}

Los sistemas de producción ganaderos, asociados al uso de pradera sin arboles son un ecosistema terrestre de gran importancia, que ocupa casi la cuarta parte de la superficie terrestre (Clough et al. 2020). La creciente demanda de alimento ha aumentado en las últimas décadas, relacionado con el crecimiento demográfico, lo cual ha conllevado a la conversión de áreas boscosas en sistemas de producción agropecuarios (FAO y GTIS 2015).

Estos sistemas son altamente dependientes de fertilizantes nitrogenados $(\mathrm{N})$ sintéticos, esenciales para la maximización de la productividad a period of 12 months (November 2014 - 2015). An experimental design of randomized complete block design was used, in an arrangement of divided plots and 2 repetitions, where main plot corresponded to grasses (Brachiaria humidicola CIAT679 and Panicum maximum cv. Tanzania) and bare soil; and the subplots fertilization dose of N $\left(0,150,300 \mathrm{~kg} \cdot \mathrm{ha}^{-1} \cdot \mathrm{year}^{-1} \mathrm{~N}\right)$. Results. The $\mathrm{N}_{2} \mathrm{O}$ fluxes fluctuated according to the soil moisture content, associated with periods of great or less precipitation in the region. Coverage with the grass Brachiaria humidicola cv CIAT679 showed lower $\mathrm{N}_{2} \mathrm{O}$ emissions, while the doses of nitrogen fertilizers increased $\mathrm{N}_{2} \mathrm{O}$ emissions. These effects were partially attenuated by the use of the coverage with Brachiaria humidicola CIAT679. Conclusions. The results obtained indicate that nitrous oxide emissions in livestock systems could vary from the type of coverage and the doses of nitrogen fertilizer used. In our case, it was found that coverage with Brachiaria humdicola CIAT769 in livestock production systems reduces nitrous oxide emissions by more than $40 \%$ with maximum $\mathrm{N}$ soil application of $300 \mathrm{~kg} \cdot \mathrm{ha}^{-1} \cdot$ year $^{-1}$.

(Lam et al. 2018). Sin embargo, las plantas rara vez son capaces de asimilar más del $50 \%$ del fertilizante nitrogenado aplicado, con la pérdida de $\mathrm{N}$ del sistema suelo - planta, por medio de la lixiviación, volatilización, nitrificación y desnitrificación, que representa una fuente directa de emisiones de gases de efecto invernadero como el óxido nitroso $-\mathrm{N}_{2} \mathrm{O}$ (Cameron et al. 2013). Este es uno de los gases de efecto invernado que, en las últimas décadas, registra las mayores tasas de acumulación en la atmósfera (Herrero et al. 2016), reportado además, como uno de los de mayor permanencia o duración ( $>100$ años), con potencial de calentamiento global (PCG), 310 veces mayor que el del dióxido de carbono $\left(\mathrm{CO}_{2}\right)$ 
(Gao et al. 2014, Byrnes et al. 2017, Liang et al. 2018). Se considera que el $\mathrm{N}_{2} \mathrm{O}$ es la sustancia que más agota o degrada la capa de ozono, por lo que se le ha dado mayor relevancia en estudios sobre su mitigación (Ravishankara et al. 2009, CATIE 2015).

Las actividades antrópicas, relacionadas con el uso en los sistemas agropecuarios de los fertilizantes nitrogenados $(\mathrm{N})$, se considera que promueven las emisiones de $\mathrm{N}_{2} \mathrm{O}$ hacia la atmosfera, lo cual se relaciona con los procesos de nitrificación y desnitrificación que ocurren continuamente en los suelos (González-Estrada y Camacho-Amador 2017). Se ha reportado que la entrada de fertilizantes nitrogenados estimula estos procesos, lo que genera a su vez un aumento de las tasas de emisión de $\mathrm{N}_{2} \mathrm{O}$ (Erickson et al. 2001, Butterbach Bahl et al. 2013).

De acuerdo con el inventario nacional de gases de efecto invernadero realizado en Colombia, se indica que el $26 \%$ de las emisiones del país se relacionan con actividades del sector agropecuario, ya que representa un total de 67,3 Mton $\mathrm{CO}_{2}$ eq.año ${ }^{-1}$, de las cuales el 12,7\% (8,55 Mton $\mathrm{CO}_{2}$ eq) están relacionadas con las emisiones ocasionadas por el sector pecuario (orina $\mathrm{y}$ estiércol de animales en pastoreo), seguido por la aplicación de fertilizantes nitrogenados sintéticos $\left(2,6 \% ; 1,75\right.$ Mton $\mathrm{CO}_{2}$ eq.año $\left.{ }^{-1}\right)$ y la gestión de suelos orgánicos drenados $(2,1 \% ; 1,41$ Mton $\mathrm{CO}_{2}$ eq.año-1), entre otros (Pulido et al. 2016, Torres, 2020). Diversas investigaciones han demostrado que el uso y manejo del nitrógeno en los sistemas de producción agropecuario es uno de los factores de mayor impacto en la emisión de gases de efecto invernadero (GEI) (Dalal et al. 2003, Bodelier y Laanbroek 2004, LeBauer y Treseder 2008). Asimismo, se ha demostrado con respecto al flujo biogénico de gases de efecto invernadero (GEI) que la magnitud y dirección de los flujos puede variar entre ecosistemas, debido a las condiciones ambientales específicas del hábitat, variaciones en la humedad del suelo y el tipo de cubierta vegetal (Neff et al. 1994, Gulledge y Schimel 2000). Debido al creciente uso de fertilizantes nitrogenados en la producción ganadera en el valle del Sinú, se consideró de gran importancia evaluar los flujos de $\mathrm{N}_{2} \mathrm{O}$ en diferentes tipos de cobertura de pasturas, frente a las distintas dosis de fertilizante nitrogenado $(\mathrm{N})$.

\section{MATERIALES Y MÉTODOS}

Sitio experimental. La investigación se realizó durante un periodo de 365 días consecutivos, con toma de información mensual de los flujos de $\mathrm{N}_{2} \mathrm{O}$ en un sistema ganadero de producción de carne, entre noviembre 2014 a noviembre 2015, bajo las condiciones del centro de investigación Turipaná, perteneciente a la Corporación Colombiana de Investigación Agropecuaria - AGROSAVIA. Este centro se encuentra localizado al norte de Colombia en la subregión Valle del Sinú municipio de Cereté Córdoba, con coordenadas geográficas $8^{\circ} 51^{\prime} 3,9^{\prime \prime}$ $\mathrm{N}$ y $75^{\circ} 48^{\prime} 3,3$ " W. El centro de investigación se encuentra a $15 \mathrm{msnm}$, temperatura promedio $28^{\circ} \mathrm{C}$, precipitación acumulativa anual de 1200 a $1300 \mathrm{~mm} \cdot \mathrm{anno}^{-1}$ y humedad relativa promedio año $82 \%$. Las precipitaciones en la región del valle medio del rio Sinú presenta comportamiento bimodal, con 2 periodos bien definidos, uno lluvioso comprendido entre abril y noviembre, y otro seco entre diciembre y marzo. Las condiciones agroecológicas predominantes son las de Bosque seco tropical (Bs T), descrita por Holdridge (2000).

El área de estudio posee suelos de planicie, correspondiente a tierras bajas, en las cuales el material de origen es sedimentario, con texturas finas. El drenaje natural es imperfecto en las áreas más altas y pobre en las áreas más bajas. Los grupos texturales predominantes son franco-arcilloso, franco-limoso y franco-arcillolimoso, con mayor presencia del primer grupo textural. El suelo bajo estudio correspondió a un Vertic Endoaquepts (Soil Survey Staff 2014), el cual presenta un régimen de humedad údico y temperatura isohipertérmico, con secuencia de horizontes Ap, Bx, Bg y Cg. 
Tratamientos. La investigación se realizó en un área que por años ha estado bajo producción ganadera. Se seleccionaron 12 ha, las cuales presentaban potreros con las pasturas Brachiaria humidícola cv. CIAT679 y Panicum maximum cv. Tanzania. Se utilizó un diseño experimental de parcelas divididas con 2 repeticiones, en donde las parcelas principales correspondieron a las coberturas con gramíneas (PP) y las subparcelas (SP) a las dosis de fertilización nitrogenada $\left(0,150\right.$ y $300 \mathrm{~kg} \cdot \mathrm{ha}^{-1} \cdot$ año $\left.^{-1}\right)$, aleatorizadas dentro de las PP. Para La fuente de nitrógeno utilizada fue la Urea $\left(\mathrm{CO}\left(\mathrm{NH}_{2}\right)_{2}\right)$, con una concentración de $46 \%$ de $\mathrm{N}$. Las aplicaciones de $\mathrm{N}$ se realizaron al voleo, en forma superficial, en 3 aplicaciones fraccionadas de la dosis total, las cuales se realizaron bajo condiciones de suelo húmedo, según el comportamiento de las precipitaciones en la región. La primera aplicación del fertilizante nitrogenado se realizó en noviembre, al final de la temporada anual de lluvias, la segunda aplicación se realizó en abril, al inicio de la temporada de lluvias del siguiente año, y la tercera aplicación se realizó al terminarse la época denominada veranillo de San Juan, a mediados de agosto. A lado de cada parcela principal se dejaron áreas de terreno con suelo descubierto. Como testigo absoluto, sobre las cuales se realizaron las aplicaciones de $\mathrm{N}$ en las mismas dosis y forma de aplicación que las anteriores (Tabla 1).

Tabla 1. Tratamientos de fertilización nitrogenada implementados para monitorear las emisiones de $\mathrm{N}_{2} \mathrm{O}$ en suelos bajo producción ganadera.

Valle Medio del Sinú, Colombia.

\begin{tabular}{|c|c|c|}
\hline Cobertura (PP) & Dosis de Nitrógeno (UREA, $\left.\mathrm{CO}\left(\mathrm{NH}_{2}\right)_{2}\right) \mathrm{kg} \cdot \mathrm{ha}^{-1} \cdot \mathrm{anno}^{-1}(\mathrm{SP})$ & Tratamientos \\
\hline \multirow{3}{*}{ Brachiaria humidicola cv. CIAT679 } & 0 & $\mathrm{Bh} \_0$ \\
\hline & 150 & Bh_150 \\
\hline & 300 & Bh_300 \\
\hline \multirow{3}{*}{ Panicum maximum cv. Tanzania } & 0 & Pm_0 \\
\hline & 150 & Pm_150 \\
\hline & 300 & Pm_300 \\
\hline \multirow{3}{*}{ Suelo descubierto } & 0 & Sd_0 \\
\hline & 150 & Sd_150 \\
\hline & 300 & Sd_300 \\
\hline
\end{tabular}

Con el anterior arreglo experimental, las PP quedaron conformadas por un área de 3 ha y las SP por un área de 1 ha. Las parcelas con suelo descubierto tuvieron un área de 0,3 ha a manera de PP y la SP de 0,1 ha.

\section{Variables evaluadas}

Ambientales. Se realizó el registro de variables ambientales como la precipitación $\left(\mathrm{mm} \cdot \mathrm{mes}^{-1}\right)$, temperaturas máxima, mínima, media diaria $\left({ }^{\circ} \mathrm{C}\right)$ y humedad relativa media $(\%)$ durante los 12 meses de evaluación, mediante la instalación de una estación climatológica (Vantage Pro2 Plus Fan, Davis Instruments), en un área anexa al experimento (Tabla 2). Los registros de las variables ambientales indicaron que, el $58 \%$ de las evaluaciones de los flujos de $\mathrm{N}_{2} \mathrm{O}$ se realizaron bajo condiciones de época seca, con precipitaciones mensuales inferiores a los 100 mm.mes ${ }^{-1}$ (Tabla 2). 
Tabla 2. Comportamiento de algunas variables ambientales en el área de estudio durante el periodo de evaluación de flujos de $\mathrm{N}_{2} \mathrm{O}$.

\begin{tabular}{cccccc}
\hline Mes & $\mathrm{T} \max \left({ }^{\circ} \mathrm{C}\right)$ & $\mathrm{T} \min \left({ }^{\circ} \mathrm{C}\right)$ & $\mathrm{T} \operatorname{med}\left({ }^{\circ} \mathrm{C}\right)$ & $\mathrm{HR}(\%)$ & $\mathrm{P}\left(\mathrm{mm} \cdot \mathrm{mes}^{-1}\right)$ \\
\hline nov-14 & 32,91 & 24,07 & 28,49 & 74,00 & 31,10 \\
dic-14 & 33,26 & 24,15 & 28,70 & 75,95 & 67,00 \\
ene-15 & 34,36 & 22,87 & 28,62 & 67,61 & 0,40 \\
feb-15 & 34,99 & 23,77 & 29,38 & 66,35 & 9,30 \\
mar-15 & 35,64 & 24,13 & 29,89 & 63,31 & 0,00 \\
abr-15 & 34,89 & 24,74 & 29,82 & 70,57 & 141,60 \\
may-15 & 33,60 & 24,46 & 29,03 & 75,06 & 217,20 \\
jun-15 & 34,67 & 25,11 & 29,89 & 72,00 & 39,20 \\
jul-15 & 33,81 & 24,31 & 29,06 & 75,24 & 220,80 \\
ago-15 & 33,90 & 24,47 & 29,18 & 76,26 & 87,80 \\
sep-15 & 33,35 & 24,63 & 28,99 & 79,59 & 91,00 \\
oct-15 & 32,78 & 24,07 & 28,42 & 79,26 & 208,00 \\
nov-15 & 32,22 & 24,45 & 28,34 & 81,13 & 135,60 \\
\hline
\end{tabular}

Tmax $=$ Temperatura máxima $\left({ }^{\circ} \mathrm{C}\right) ;$ Tmin $=$ Temperatura mínima $\left({ }^{\circ} \mathrm{C}\right) ;$ Tmed $=$ Temperatura media $\left({ }^{\circ} \mathrm{C}\right)$; $\mathrm{HR}=$ Humedad Relativa $(\%) ; \mathrm{P}=$ Precipitación $(\mathrm{mm})$.

Propiedades de los suelos. Para la determinación de las propiedades físicas del sitio experimental, se tomaron muestras al inicio y al final del experimento en cada subparcela, a una profundidad de 0,20 $\mathrm{m}$. La determinación de la densidad aparente (Da) se realizó mediante el método del cilindro de volumen conocido $(98,17$ $\left.\mathrm{cm}^{-3}\right): \mathrm{Da}=\mathrm{Mss} / \mathrm{Vc}$, donde Mss $=$ masa de suelo seco $(\mathrm{g})$ a $105^{\circ} \mathrm{C}$ por $24 \mathrm{hr}$ y $\mathrm{Vc}=$ volumen del cilindro $\left(\mathrm{cm}^{-3}\right)$ (Burt 2014); la densidad real (Dr) mediante el método del picnómetro (Burt 2014); la porosidad total $(\mathrm{Pt})$ mediante la relación $\mathrm{Pt}=$ (1- (Da/Dr)) x 100 (Bernabé y Maineult 2015); la textura por el método de Bouyoucos y la estabilidad de agregados mediante el método del yoder modificado. El porcentaje de la porosidad ocupada por agua (water filled porosity space, WFPS) se determinó con la metodología utilizada por Contreras-Santos et al. (2021), mediante la relación:

$$
\text { WFPS }=(\text { Hum X Da / Pt }) \text { X } 100
$$

Donde

$$
\begin{aligned}
& \text { Hum }=\text { humedad gravimétrica }\left(\mathrm{g} \cdot \mathrm{g}^{-1}\right) \\
& \mathrm{Da}=\text { densidad aparente }\left(\mathrm{g} \cdot \mathrm{cm}^{-3}\right) \\
& \mathrm{Pt}=\text { porosidad total }\left(\mathrm{g} \cdot \mathrm{g}^{-1}\right)
\end{aligned}
$$

Todos los análisis físicos se realizaron a partir de los protocolos establecidos por IGAC (2006).

Para la determinación de las propiedades químicas del sitio experimental, se colectaron muestras por triplicado a una profundidad de $0,20 \mathrm{~m}$, en cada una de las subparcelas, las cuales se homogenizaron para obtener $1 \mathrm{~kg}$ de suelo por subparcela. Las muestras fueron analizadas en 
el laboratorio de Química Analítica de AGROSAVIA, con base a las metodologías propuestas por IGAC (2006). La reacción del suelo o pH, se determinó mediante el método potenciométrico, con relación 1:1 Peso/Volumen (Hendershot et al. 2007), Norma Técnica Colombiana - NTC 5264, materia orgánica $(\mathrm{MO}, \%)$ por oxidación Walkley - Black (Nelson y Sommers 1983), fósforo (P, mg. $\mathrm{kg}^{-1}$ ) por extracción Bray II modificado y cuantificación por reducción con ácido ascórbico (Bray y Kurtz 1945), Azufre (S, mg.kg-1) extracción con monofosfato de calcio $0,008 \mathrm{M}$ y cuantificación turbidimetrica, potasio; Calcio; Magnesio y sodio (K; Ca; $\mathrm{Mg}$; $\mathrm{Na}, \mathrm{cmol}(+) \cdot \mathrm{kg}^{-1}$ ) por medio espectrofotometría de absorción $\mathrm{y}$ emisión atómica (Hendershot et al. 2007, Chapman 2016), Norma Técnica Colombiana - NTC 5349, Hierro; Manganeso; Zinc y Cobre (Fe; Mn; $\mathrm{Zn} ; \mathrm{Cu}, \mathrm{mg} \cdot \mathrm{kg}^{-1}$ ) se determinaron por medio del método de Olsen modificado espectrofotometría y cuantificación por absorción atómica (NTC 5526:2007) y Boro (B, mg. $\mathrm{kg}^{-1}$ ) extracción con fosfato monobásico de calcio - Azometina $\mathrm{H}$ (NTC 5404), se realizó análisis de salinidad y alcalinidad del suelo (Bases solubles: $\mathrm{Ca}^{++}$, $\mathrm{Mg}^{++}, \mathrm{K}^{+}$y Na${ }^{+}$); Sulfatos $\left(\mathrm{SO}_{4}\right)$; Cloruros (Cl-) y bicarbonatos $\left(\mathrm{HCO}_{3}\right)$ siguiendo la metodología descrita por IGAC (2006).

Flujos de óxido nitroso $\left(\mathbf{N}_{2} \mathbf{O}\right)$. Los flujos de $\mathrm{N}_{2} \mathrm{O}$ se realizaron con la metodología de cámara cerrada estática de PVC descrita por Espinosa-Carvajal et al. (2020). La cámara constaba de 2 cilindros de $\mathrm{PVC}$, de $25 \mathrm{~cm}$ de diámetro, unidos por medio de una banda elástica de caucho (polímero elástico). El cilindro inferior abierto por ambas caras y biselado en uno de sus lados tenía una altura total de $7,5 \mathrm{~cm}$, el cual se insertó en el suelo 24 horas antes de iniciar el proceso de evaluación, a una profundidad de 5,5 $\mathrm{cm}$. El cilindro superior tenía una altura de $10 \mathrm{~cm}$ y en este caso, se instalaba al momento de iniciar las evaluaciones de flujos de $\mathrm{N}_{2} \mathrm{O}$. Este cilindro estaba cerrado por una de sus caras (superior), con la finalidad de asegurar hermetismo dentro de la cámara (microclima), con 2 agujeros tapados con material de goma, uno para introducir el termómetro para evaluar temperatura interna de la cámara y otro para tomar la muestra de gases. Las cámaras se instalaron por triplicado en cada una de las parcelas experimentales.

Las determinaciones de los flujos de $\mathrm{N}_{2} \mathrm{O}$ se realizaron a las 10:00 am, para muestras de $20 \mathrm{~mL}$ del aire dentro de la cámara a los 0 (antes de cerrar la cámara), 15, 30 y 45 min después de cerrada la cámara. De las muestras obtenidas con jeringa se descartaron los primeros $5 \mathrm{~mL}$ y el resto fue depositado en viales al vacío con capacidad de $15 \mathrm{~mL}$. Las muestras fueron rotuladas, selladas y almacenadas a temperatura ambiente bajo condiciones de oscuridad (Rondon, 2000). Las muestras fueron enviadas al laboratorio de servicios ambientales del Centro Internacional de Agricultura Tropical - CIAT (Palmira, Colombia), en donde se determinaron en cada tratamiento, las concentraciones de $\mathrm{N}_{2} \mathrm{O}$ a los tiempos $0,15,30$ y 45 min después de cerrada la cámara, mediante cromatografía de gases.

Los flujos de $\mathrm{N}_{2} \mathrm{O}$ se calcularon en función del área y tiempo, mediante la siguiente relación (Capa 2015):

$$
\begin{aligned}
& \mathrm{F}=\frac{d x}{d t} \times \frac{V \times M}{A \times V m} \\
& \text { Donde }
\end{aligned}
$$

$$
\begin{aligned}
\mathrm{F}= & \text { flujo de } \mathrm{N}_{2} \mathrm{O}\left(\mu \mathrm{g} \mathrm{N}-\mathrm{N}_{2} \mathrm{O} \mathrm{m}^{-2} \mathrm{hr}^{-1}\right) \\
\mathrm{dx} / \mathrm{dt}= & \text { pendiente de la variación de las lecturas, } \\
& \text { a los tiempos } 0,15,30 \mathrm{y} 45 \text { min después } \\
& \text { de cerrada la cámara (ppm min-1) } \\
\mathrm{V}= & \text { volumen ocupado por el gas }(\mathrm{L}) \\
\mathrm{M}= & \text { masa molar de } \mathrm{N} \text { por cada Mol de } \mathrm{N}_{2} \mathrm{O} \\
\mathrm{A}= & \text { área de la cámara }\left(\mathrm{m}^{2}\right) \\
\mathrm{Vm}= & \text { volumen molar del gas }
\end{aligned}
$$

Para obtener $\mathrm{dx} / \mathrm{dt}$ se ajustaron modelos de regresión lineales para cada cámara y muestreo realizado, para lo cual se consideraron ajustes con $R^{2}>0,90$ y $p<0,05$.

Análisis estadístico. Se realizaron análisis de varianza para comparar los flujos $\mathrm{N}_{2} \mathrm{O}$ 
entre tratamientos, con un nivel de significancia $\alpha=0,05$, junto con modelos lineales generales mixtos. Para evaluar el cumplimiento de los requisitos de normalidad y homogeneidad se utilizaron gráficos de gg, histogramas y gráficos de cajas de residuos. La homogeneidad se evaluó mediante gráficos de residuos versus datos esperados. Se seleccionó el modelo con el menor Akaike (AIC) y criterio de información bayesiano (BIC) y la máxima relación de probabilidad de registro. Para realizar la comparación de medias entre tratamientos, se utilizó la prueba HSD de Tukey y contrastes ortogonales para comparar combinaciones entre tratamientos. Todos los análisis estadísticos se realizaron en el programa estadístico SAS Enterprise Guide 8.3. Las variables ambientales y edáficas se sometieron a un análisis de correlación de Pearson y componentes principales mediante el procedimiento PROC FACTOR, que determinaron la asociación de variables frente a los flujos $\mathrm{N}_{2} \mathrm{O}$.

\section{RESULTADOS}

Propiedades del suelo. Los resultados mostraron que las propiedades físicas y químicas del suelo antes y después de concluido el periodo experimental eran similares $(p>0,05)$ entre tratamientos. La densidad aparente presentó un promedio general de $1,10 \pm 0,07 \mathrm{~g} . \mathrm{cm}^{-3}$, los valores de porosidad total estuvieron alrededor del $50 \%$. El pH mostró una media general de $6,55 \pm 0,28$; el contenido de materia orgánica (MO) expuso valores superiores a 3\%, el contenido de azufre (S) presentó valor medio de 24,48 $\pm 26,99$ mg. $\mathrm{kg}^{-1}$, el fosforo intercambiable $(\mathrm{P})$, presentó valores inferiores a $20 \mathrm{mg} \cdot \mathrm{kg}^{-1}$, el contenido de bases de intercambio (calcio, magnesio y potasio), mostró valores promedios de $13,08 \pm 2,77,12,21 \pm 1,85$ y $0,69 \pm 0,36 \mathrm{cmol} \cdot \mathrm{kg}^{-1}$, respectivamente. Los microelementos (hierro, cobre, zinc y manganeso) mostraron valores promedios de $35,60 \pm 28,86$, $1,80 \pm 0,71,3,42 \pm 0,94$ y $25,60 \pm 14,34$ mg.kg ${ }^{-1}$, respectivamente (Tabla 3 ). 


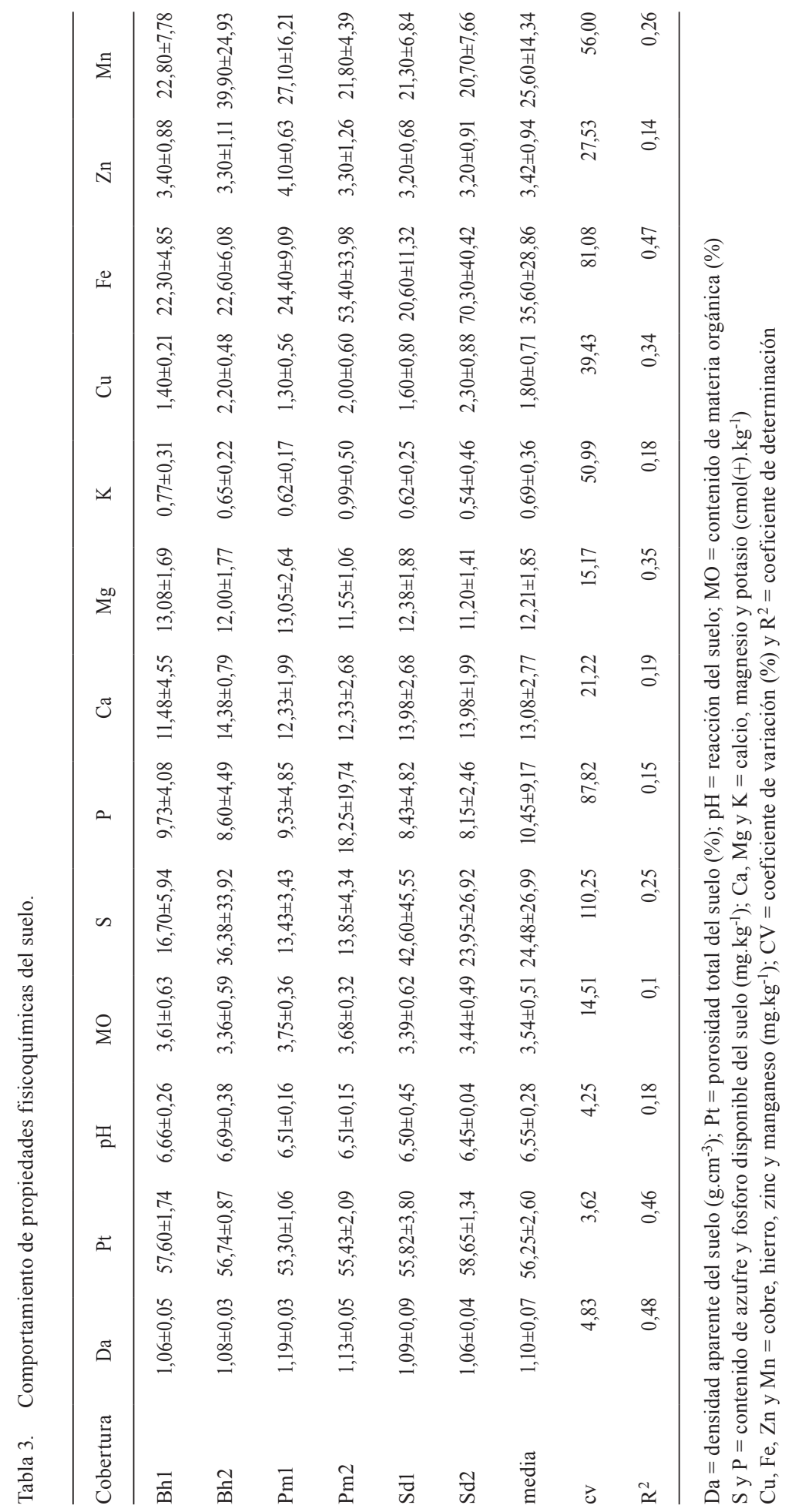


El comportamiento de la WFPS mostró variaciones asociadas al periodo de evaluación (Figura 1), donde los menores valores se presentaron en las épocas asociadas a bajas precipitaciones (diciembre de 2014 a abril de 2015). A partir de mayo, con el inicio de la temporada de lluvias, los valores de WFPS aumentaron, con valores que fluctuaron entre 55,55 y $57,57 \%$. En julio (2015) se presentó una época de bajas precipitaciones, correspondiente a un periodo conocido en la región como Veranillo de San Juan, con lo cual los valores de WFPS disminuyeron, pero a partir del mes de agosto, con el reinicio de las precipitaciones, la WFPS mantuvo valores superiores al $60 \%$, hasta final del periodo experimental (Figura 1).

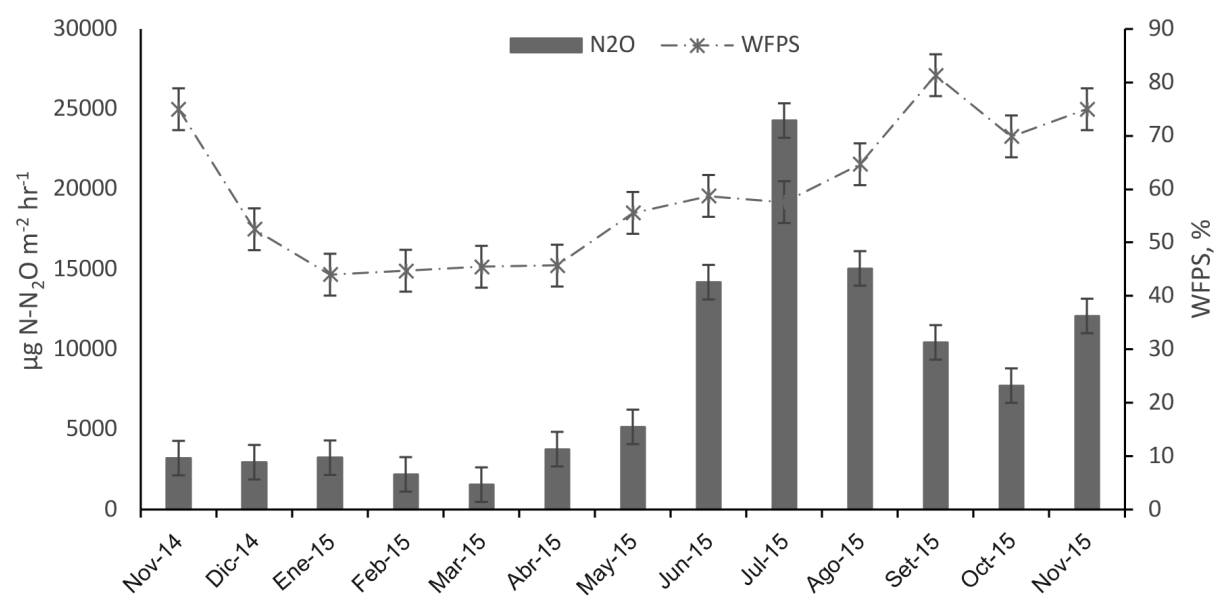

Figura 1. Comportamiento de los flujos de óxido nitroso $\left(\mathrm{N}_{2} \mathrm{O}\right)$ durante el periodo de 12 meses de evaluaciones. C.I Turipaná, Cereté - Córdoba. 2015.

Flujos de Óxido nitroso $\left(\mathrm{N}_{2} \mathrm{O}\right)$. Las dinámicas de los flujos de $\mathrm{N}_{2} \mathrm{O}$ mostraron una fuerte relación $(\mathrm{p}<0,0001)$ en relación con las variaciones en las precipitaciones. Al inicio del periodo experimental, en periodos de bajas precipitaciones, entre noviembre de 2014 hasta mayo de 2015, los flujos de $\mathrm{N}_{2} \mathrm{O}$ alanzaron los menores valores promedios de emisión, con un promedio de $3154,2 \mu \mathrm{g} \mathrm{N}-\mathrm{N}_{2} \mathrm{O} \mathrm{m}^{-2} \mathrm{~h}^{-1}$ y un rango de variación entre 1554,4 a $5159,9 \mu \mathrm{g} \mathrm{N}_{-} \mathrm{N}_{2} \mathrm{O} \mathrm{m} \mathrm{m}^{-2} \mathrm{~h}^{-1}$, siendo marzo (2015) el de menor emisión (Figura 1). Asimismo, se observó que, con el inicio de la temporada de lluvias, los flujos de $\mathrm{N}_{2} \mathrm{O}$ aumentaron, registrándose los mayores valores entre junio a agosto 2015, siendo julio de 2015 donde se observó el pico máximo de emisiones, con valores que alcanzaron los 24284,3 $\mu \mathrm{g} \mathrm{N}-\mathrm{N}_{2} \mathrm{O} \mathrm{m}^{-2} \mathrm{~h}^{-1}$.

En relación con los tratamientos evaluados se observó una interacción significativa $(\mathrm{p}<0,05)$, entre los flujos de $\mathrm{N}_{2} \mathrm{O}$ y las dosis de nitrógeno y la cobertura utilizada. En el caso de la pastura Brachiaria humidicola cv. CIAT679, los flujos de $\mathrm{N}_{2} \mathrm{O}$ aumentaron con el incremento en la dosis de fertilización $\mathrm{N}$, de $2000 \mu \mathrm{g}$ $\mathrm{N}-\mathrm{N}_{2} \mathrm{O} \mathrm{m}^{-2} \mathrm{~h}^{-1}$ con $0 \mathrm{~kg} \cdot \mathrm{ha}^{-1} \cdot \mathrm{año}^{-1} \mathrm{~N}$ a $8000 \mu \mathrm{g}$ $\mathrm{N}-\mathrm{N}_{2} \mathrm{O} \mathrm{m} \mathrm{m}^{-2} \mathrm{~h}^{-1}$ con $300 \mathrm{~kg} \cdot \mathrm{ha}^{-1}$.año ${ }^{-1} \mathrm{~N}$ (Figura 2). Un comportamiento similar se observó en la pastura Panicum maximum cv. Tanzania en donde el incremento en las emisiones de $\mathrm{N}_{2} \mathrm{O}$ fueron de 2,0 a 2,7 veces superior, al aplicar las dosis superiores de nitrógeno al suelo (150 y 300 $\mathrm{kg} \cdot \mathrm{ha}^{-1} \cdot \mathrm{año} \mathrm{o}^{-1} \mathrm{~N}$ ). En los suelos suelo sin cobertura de pasturas, los flujos de $\mathrm{N}_{2} \mathrm{O}$ fueron superiores a los suelos con cobertura e igualmente se incrementaron con el aumento en las dosis de fertilización nitrogenada, de 8000 a $14000 \mu \mathrm{g}$ $\mathrm{N}-\mathrm{N}_{2} \mathrm{O} \mathrm{m} \mathrm{m}^{-2} \mathrm{~h}^{-1}$ con las dosificaciones de $150 \mathrm{y}$ $300 \mathrm{~kg} \cdot \mathrm{ha}^{-1} \mathrm{~N}$. 


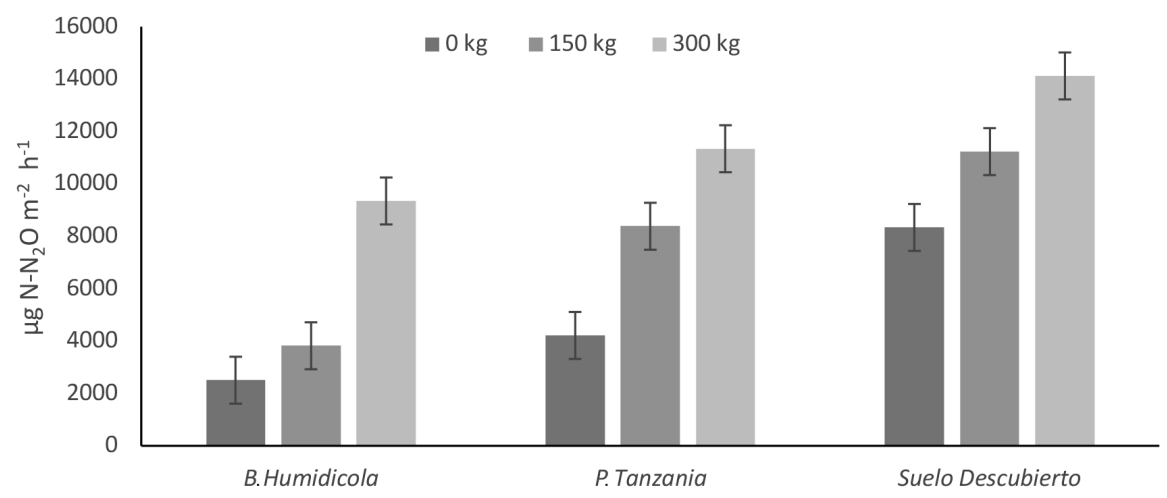

Figura 2. Comportamiento de los flujos de $\mathrm{N}_{2} \mathrm{O}$ bajo diferentes dosis de fertilización nitrogenada en 3 tipos de cobertura. C.I Turipaná, Cereté - Córdoba. 2015.

Los contrastes ortogonales permitieron comparar combinaciones entre tratamientos, que permitieron determinar que, bajo la misma dosis de fertilización nitrogenada $\left(150 \mathrm{~kg} \cdot \mathrm{ha}^{-1} \cdot \mathrm{año}^{-1}\right.$ $\mathrm{N})$, los flujos de $\mathrm{N}_{2} \mathrm{O}$ fueron inferiores en la pastura Brachiaria humidicola cv. CIAT679 que en Panicum maximum cv. Tanzania. Con valores promedios de $3819 \mu \mathrm{g} \mathrm{N}-\mathrm{N}_{2} \mathrm{O} \mathrm{m} \mathrm{m}^{-2} \mathrm{~h}^{-1}$ y $8381,7 \mu \mathrm{g}$ $\mathrm{N}-\mathrm{N}_{2} \mathrm{O} \mathrm{m} \mathrm{m}^{-2} \mathrm{~h}^{-1}$, respectivamente. La comparación de la cobertura con pasturas, en relación con el suelo descubierto, a una dosis de 150 kg.ha-1, mostraron que bajo suelo descubierto las emisiones de $\mathrm{N}_{2} \mathrm{O}$ se triplicaron, y alcanzaron un promedio de $11230,4 \mu \mathrm{g} \mathrm{N}-\mathrm{N}_{2} \mathrm{O} \mathrm{m} \mathrm{m}^{-2} \mathrm{~h}^{-1}$. Igual comportamiento se detectó, bajo la dosis de 300 kg.ha $a^{-1}$ año ${ }^{-1} \mathrm{~N}$ (Figura 2).

Correlaciones. Los análisis de correlación corroboraron la relación significativa $(\mathrm{p}<0,05)$ entre los flujos de $\mathrm{N}_{2} \mathrm{O}$ y el comportamiento de las variables ambientales y las propiedades fisicoquímicas del suelo. Estos análisis mostraron una correlación positiva y significativa $(\mathrm{r}=0,54)$ entre los flujos de $\mathrm{N}_{2} \mathrm{O}$ y la ocurrencia de las precipitaciones. De igual forma, se observó una correlación positiva y significativa de los flujos con la humedad relativa $(r=0,43)$ e inversa con la temperatura ambiental $(\mathrm{r}=$ 0,38). Por otra parte, el espacio poroso ocupado por agua (WFPS) mostró correlación positiva $(\mathrm{r}=0,53)$, por los efectos directos de la humedad del suelo sobre los flujos de este gas. Igualmente, se encontraron correlaciones significativas $(\mathrm{p}<0,05)$ pero débiles entre algunas variables químicas de suelo, tales como el $\mathrm{pH}(\mathrm{r}=-0,15)$, azufre en el suelo $(\mathrm{r}=0,14)$, potasio intercambiable $(\mathrm{r}=-0,18)$, hierro disponible $(\mathrm{r}=0,11)$ y contenido de sulfatos $(\mathrm{r}=0,22)$ (Tabla 4). Lo anterior indica que valores altos o bajos de estas variables pueden generar aumentos o disminuciones menores en las tasas de emisión de $\mathrm{N}_{2} \mathrm{O}$ hacia la atmósfera. 
Tabla 4. Análisis de correlación de Pearson de variables ambientales, edáficas y flujos de $\mathrm{N}_{2} \mathrm{O}$ bajo el sistema de producción intensivo ganadero.

\begin{tabular}{|c|c|c|c|c|c|c|c|c|c|c|c|c|c|}
\hline Variables & W & $\mathrm{Pt}$ & WFPS & Temp & Prec & HR & $\mathrm{pH}$ & $\mathrm{S}$ & $\mathrm{Mg}$ & K & $\mathrm{Fe}$ & SO4 & $\mathrm{CE}$ \\
\hline $\mathrm{r}$ & 0,49 & 0,14 & 0,53 & $-0,38$ & 0,54 & 0,43 & $-0,15$ & 0,14 & 0,11 & $-0,18$ & 0,11 & 0,22 & 0,1 \\
\hline $\mathrm{N}_{2} \mathrm{O}$ & $* *$ & $*$ & $* *$ & $* *$ & $* *$ & $* *$ & $*$ & $*$ & ns & $*$ & $*$ & $* *$ & $\mathrm{~ns}$ \\
\hline
\end{tabular}

$\mathrm{N}_{2} \mathrm{O}=$ Flujos de óxido nitroso $\left(\mu \mathrm{g} \mathrm{N}-\mathrm{N} 2 \mathrm{O} \mathrm{m} \mathrm{m}^{-2} \cdot \mathrm{h}^{-1}\right)$; Prec = precipitación acumulada $\left(\mathrm{mm} \cdot \mathrm{mes}^{-1}\right)$, Temp = temperatura media ambiental $\left({ }^{\circ} \mathrm{C}\right) ; \mathrm{HR}=$ humedad relativa media $(\%) ; \mathrm{W}=$ humedad gravimétrica $\left(\mathrm{g} \cdot \mathrm{g}^{-1}\right) ; \mathrm{Pt}=$ porosidad total del suelo $(\%)$, WFPS $=$ porcentaje de poros llenos de agua (\%); $\mathrm{pH}=$ concentración de iones $\mathrm{H}^{+}$o reacción de la solución del suelo, $\mathrm{S}=$ contenido de azufre disponible en el suelo $\left(\mathrm{mg} \cdot \mathrm{kg}^{-1}\right), \mathrm{Mg}=$ contenido de magnesio disponible en el suelo ( $\left.\mathrm{cmol}(+) \cdot \mathrm{kg}^{-1}\right) ; \mathrm{K}=$ contenido de potasio disponible en el suelo $\left(\mathrm{cmol}(+) \cdot \mathrm{kg}^{-1}\right) ; \mathrm{Fe}=$ hierro disponible en el suelo $\left(\mathrm{mg} \cdot \mathrm{kg}^{-1}\right) ; \mathrm{SO}_{4}=$ Sulfatos $\left(\mathrm{meq} \cdot \mathrm{L}^{-1}\right) ; \mathrm{CE}=$ conductividad eléctrica $\left(\mathrm{dS} . \mathrm{m}^{-1}\right) ; \mathrm{r}=$ coeficientes de correlación de Pearson $(\alpha=0,05) ;{ }^{*} \mathrm{p}<0,01$ significativo; $* *=\mathrm{p}<0,0001$ altamente significativo y $\mathrm{ns}=\mathrm{p}>0,05$ no significativo.

El análisis de componentes principales (Tabla 5) mostró que 4 autovectores obtuvieron valores superiores a uno $(>1)$, capaces de explicar el $80 \%$ las emisiones de $\mathrm{N}_{2} \mathrm{O}$ generadas dentro de las coberturas. En este caso, el componente uno (CP 1) fue capaz de explicar el $32 \%$ de la varianza, componente que estuvo dominado por las variables espacio poroso lleno de agua $(0,95)$, contenido de humedad del suelo $(0,86)$ y la precipitación $(0,82)$. El componente 2 (CP 2), fue capaz de explicar el 25,18\% de la varianza, lo que resalta los vectores relacionados con el contenido de sulfatos $(0,96)$, el contenido de magnesio disponible $(0,94)$ y el contenido de azufre del suelo $(0,90)$. El componente 3 (CP 3) fue capaz de explicar el 12,79\%, y estuvo dominado por las variables climáticas temperatura ambiental $(0,89)$ y humedad relativa $(0,81)$. El componente 4 (CP 4$)$, fue capaz de explicar el $10,73 \%$ de los flujos de $\mathrm{N}_{2} \mathrm{O}$, y mostró dominancia por la variable física de suelo porosidad total $(-0,87)$. 
Tabla 5. Análisis de componentes principales de variables que mejor explican el comportamiento de los flujos de $\mathrm{N}_{2} \mathrm{O}$ en el suelo.

\begin{tabular}{|c|c|c|c|c|c|}
\hline \multicolumn{6}{|c|}{ Componentes principales } \\
\hline & CP 1 & CP 2 & CP 3 & CP 4 & \\
\hline Autovalor & 6,5 & 4,5 & 2,1 & 1,7 & \\
\hline Varianza (\%) & 32,08 & 25,18 & 12,79 & 10,73 & \\
\hline Acumulada (\%) & 32,08 & 57,27 & 70,05 & 80,78 & \\
\hline Indicador $^{\beta}$ & \multicolumn{4}{|c|}{ Vectores propios $^{+}$} & Comunalidades \\
\hline $\mathrm{N}_{2} \mathrm{O}$ & 0,66 & 0,13 & $-0,37$ & 0,06 & 0,60 \\
\hline Prec & 0,82 & 0,03 & $-0,20$ & 0,06 & 0,72 \\
\hline Temp & $-0,07$ & 0,04 & 0,89 & $-0,12$ & 0,82 \\
\hline $\mathrm{HR}$ & 0,42 & $-0,04$ & $-0,81$ & $-0,12$ & 0,85 \\
\hline W & 0,86 & 0,07 & $-0,07$ & $-0,39$ & 0,89 \\
\hline $\mathrm{Pt}$ & 0,09 & $-0,25$ & 0,15 & $-0,87$ & 0,85 \\
\hline WFPS & 0,95 & $-0,04$ & $-0,01$ & $-0,02$ & 0,91 \\
\hline $\mathrm{pH}$ & $-0,17$ & 0,03 & $-0,18$ & 0,69 & 0,53 \\
\hline S & 0,01 & 0,90 & $-0,04$ & $-0,21$ & 0,85 \\
\hline $\mathrm{Mg}$ & 0,05 & 0,94 & 0,07 & 0,08 & 0,90 \\
\hline $\mathrm{SO}_{4}$ & 0,06 & 0,96 & 0,02 & $-0,11$ & 0,94 \\
\hline
\end{tabular}

$\beta$ Indicadores fueron descritos en la Tabla 2 .

+ Valores resaltados en negrita hacen referencia a variables que mejor explican el comportamiento del flujo de $\mathrm{N}_{2} \mathrm{O}$ en el suelo.

\section{DISCUSIÓN}

La principal variable que en mayor medida afectó los flujos de $\mathrm{N}_{2} \mathrm{O}$ al medio ambiente durante el periodo experimental estuvo relacionada con las condiciones ambientales, especialmente la precipitación y en el suelo por el espacio poroso ocupado por el agua. Lo anterior debido a los efectos del agua y la disponibilidad de oxígeno en el suelo sobre los procesos de nitrificación y denitrificación que ocurren permanentemente y de forma continua en el suelo. En este experimento, los picos más altos de emisiones de $\mathrm{N}_{2} \mathrm{O}$ hacia la atmosfera ocurrieron durante los periodos de mayor precipitación, y acumulación de agua en el suelo. Estos resultados coinciden con reportes en la literatura en los que se relacionan el comportamiento de la temporada de lluvias con el contenido de humedad en el suelo y los flujos de $\mathrm{N}_{2} \mathrm{O}$ (Ussiri y Lal 2012, De Carvalho et al. 2017, Montenegro 2020). En este caso, las fluctuaciones del agua en el suelo estuvieron representadas por la WFPS, en donde los monitoreos en todos los casos mostraron valores inferiores al 60\%, que indicaron suficientes condiciones de aireación en el suelo. Sin embargo, la dinámica de los procesos de nitrificación y desnitrificación ocurre permanentemente en el suelo, que provocan la formación o liberación de $\mathrm{N}_{2} \mathrm{O}$ hacia la atmósfera (Rivera et al. 2018, Montenegro 2020). Las mayores liberaciones de $\mathrm{N}_{2} \mathrm{O}$ en este experimento se generaron de julio a noviembre (2015), en los cuales se registraron 
los mayores valores de precipitación, asociados a mayor tasa de acumulación de humedad edáfica. Los suelos del valle medio del Sinú prestan drenaje imperfecto que ocasiona que, en periodos de precipitaciones abundantes, se encharquen y demoren en la evacuación de los excesos de agua.

En relación con las coberturas, se resalta el comportamiento de la pastura Brachiaria humidicola cv. CIAT679 en la cual se registraron menores valores de emisiones de $\mathrm{N}_{2} \mathrm{O}$, aun a altas dosis de fertilización nitrogenada $(150$ y 300 $\mathrm{kg} \cdot \mathrm{ha}^{-1}$ ). Estos resultados resaltan la importancia de las coberturas vegetales en la reducción de las emisiones de $\mathrm{N}_{2} \mathrm{O}$ hacia la atmosfera, lo cual ha sido corroborado por otras personas autoras, en sistemas de producción ganadera y de cultivos (Singh et al. 2020). La capacidad de Brachiaria humidicola $\mathrm{cv}$. CIAT679 para mostrar menores emisiones de $\mathrm{N}_{2} \mathrm{O}$, probablemente se relacionan con la liberación de metabolitos secundarios a la rizosfera (braquialactonas), que afectan la actividad microbial y los procesos de nitrificación y desnitrificación en el suelo (Pastrana et al. 2011, Byrnes et al. 2017, Simon et al. 2020, Nakamura et al. 2020).

La aplicación de fertilizantes nitrogenados amplia la disponibilidad de $\mathrm{N}$ en el suelo, lo que ocasionó, invariablemente mayores flujos de $\mathrm{N}_{2} \mathrm{O}$ hacia la atmósfera (Dobbie et al. 1999, De Carvalho et al. 2017, Bastidas et al. (2020). En este caso, las coberturas vegetales reducen los impactos ambientales negativos que estas aplicaciones generan. Para nuestro caso la pastura Brachiaria humidicola cv. CIAT679, mostró una reducción del $33 \%$ en las emisiones $\left(9347,1 \mu \mathrm{g} \mathrm{N}-\mathrm{N}_{2} \mathrm{O} \mathrm{m}{ }^{-2}\right.$ $\mathrm{h}^{-1}$ ), comparado con las emisiones en suelo descubierto (14121 $\mu \mathrm{g} \mathrm{N}-\mathrm{N}_{2} \mathrm{O} \mathrm{m}^{-2} \mathrm{~h}^{-1}$ ), bajo dosis de aplicación superiores a $150 \mathrm{~kg} \cdot \mathrm{ha}^{-1} \cdot \mathrm{anno}^{-1} \mathrm{~N}$.

\section{CONCLUSIONES}

La tendencia creciente de fertilización de las pasturas con fuentes nitrogenadas para aumentar la disponibilidad de forraje aumenta el riesgo de generar altas cantidades de $\mathrm{N}_{2} \mathrm{O}$ a la atmósfera. Sin embargo, el uso de coberturas con gramíneas puede atenuar en parte estos efectos, en donde diferentes especies o cultivares pueden ejercer una mejor función. Según estos resultados, la pastura Brachiaria humidicola cv CIAT679 establecida en los sistemas de producción ganaderos en el Valle Medio del Sinú, podría contribuir en mayor medida en reducir las emisiones de óxido nitrosos en más del $40 \%$, frente a otras pasturas de corriente uso en la región.

\section{AGRADECIMIENTOS}

Las personas autoras expresan su gratitud con la Corporación Colombiana de Investigación Agropecuaria (AGROSAVIA) y el Ministerio de Agricultura y Desarrollo Rural (MADR) por financiar el proyecto titulado "Flujos De Gases De Efecto Invernadero En Sistemas Intensivos De Producción De Carne En Suelos Del Valle Medio Del Sinú “ generado como opción de grado de maestría, derivado del proyecto "Producción intensiva de carne en pasturas con diferente capacidad para inhibir la nitrificación y reducir las emisiones de gases de efecto invernadero" bajo de la cual se obtuvo este documento.

\section{LITERATURA CITADA}

Bastidas, M; Villegas, D; Ruden, A; Mazabel, J; Enciso, K; Gutierrez, JF; Arango, J. 2020. Determinación de la eficiencia en el uso del nitrógeno (UEN) por principales pasturas forrajeras de trópico bajo en respuesta a diferentes fertilizantes nitrogenados - Informe final de resultados. Palmira, Colombia, Alianza Bioversity International - CIAT. 79 p.

Bernabé, Y; Maineult, A. 2015. Physics of porous media: fluid Flux through porous media. In Schubert, G (ed.) Treatise on Geophysics. 2 ed. Vol 11. Resources in near-surface earth. Elsevier, Amsterdam, NLD. p.19-41. DOI: 10.1016/b978-0-444-53802-4.00188-3.

Bodelier, PLE; Laanbroek, HJ. 2004. Nitrogen as a regulatory factor of methane oxidation in soils and sediments. FEMS Microbiol. Ecol. 47:265-277.

Burt, R. 2014. Soil survey field and laboratory methods manual. Soil Survey Investigations Report No. 51, Version 2. WA, USA, US Department of Agriculture, Natural Resources Conservation Service. s.p. 
Butterbach-Bahl, K; Baggs, EM; Dannenmann, M; Kiese, R; Zechmeister-Boltenstern, S. 2013. Nitrous oxide emissions from soils: how well do we understand the processes and their controls? Philosophical Transactions of the Royal Society B: Biological Sciences 368(1621):20130122. DOI:10.1098/ rstb.2013.0122.

Bray, RH; Kurtz, LT. 1945. Determination of total, organic, and available forms of phosphorus in soils. Soil Science 59(1):39-46. DOI: 10.1097/00010694194501000-00006.

Byrnes, RC; Nùñez, L; Arenas, J; Rao, I; Trujillo, C; Alvarez, C; Arango, J; Rasche, F; Chirinda, N. 2017. Biological nitrification inhibition by Brachiaria grasses mitigates soil nitrous oxide emissions from bovine urine patches. Soil Biol. Biochem. 107:156163. DOI: 10.1016/j.soilbio.2016.12.029.

Cameron, KC; Di, HJ; Moir, JL. 2013. Nitrogen losses from the soil/plant system: a review. Annals of Applied Biology 162(2):145-173. DOI: 10.1111/aab.12014.

Capa, MED. 2015. Efecto de la fertilización orgánica y mineral en las propiedades del suelo, la emisión de los principales gases de efecto invernadero y en las diferentes fases fenológicas del cultivo de café (Coffea arabica L.). Tesis Ph.D. Madrid, España, Universidad de Madrid. 190 p.

CATIE (Centro Agronómico Tropical de Investigación y Enseñanza). 2015. Medición de óxido nitroso en pasturas de Costa Rica (en línea). Costa Rica. Consultado 12 set. 2021. Disponible en https://www. catie.ac.cr/catie- noticias/943- miden-oxido-nitrosoen-pasturas-de-costa-rica.html

Chapman, HD. 2016. Cation-exchange capacity. Methods of Soil Analysis. American Society of Agronomy. Wisconsin, USA, American Society of Agronomy. p. 891-901. DOI: 10.2134/agronmonogr9.2.c6.

Clough, TJ; Cardenas, LM; Friedl, J; Wolf, B. 2020. Nitrous oxide emissions from ruminant urine: science and mitigation for intensively managed perennial pastures. Current Opinion in Environmental Sustainability 47:21-27. DOI: $10.1016 / \mathrm{j}$. cosust.2020.07.001.

Contreras-Santos, J; Martinez-Atencia, J; Raghavan, B; López-Rebolledo, L; Garrido-Pineda, J. 2021. Silvopastoral systems: Mitigation of greenhouse gases in the Tropical Dry Forest - Colombia. Agronomía Mesoamericana 32(3):901-919. DOI: 10.15517/am.v32i3.43313.

Dalal, RC; Wang, WJ; Robertson, GP; Parton, WJ. 2003. Nitrous oxide emission from Australian agricultural lands and mitigation options: a review. Aust. J. Soil Res. 41:165-195.

De Carvalho, AM; de Oliveira, WRD; Ramos, MLG; Coser, TR; de Oliveira, AD; Pulrolnik, K; Souza, KW; Vileta, L; Marchão, RL. 2017. Soil $\mathrm{N}_{2} \mathrm{O}$ fluxes in integrated production systems, continuous pasture and Cerrado. Nutr. Cycl. Agroecosystems 108(1):6983. DOI: $10.1007 / \mathrm{s} 10705-017-9823-4$.

Dobbie, KE; McTaggart, IP; Smith, KA. 1999. Nitrous oxide emissions from intensive agricultural systems: Variations between crops and seasons, key driving variables, and mean emission factors. J. Geophys. Res. Atmos. 104(D21):26891-26899. DOI: 10.1029/1999jd900378.

Erickson, H; Keller, M; Davidson, EA. 2001. Nitrogen oxide fluxes and nitrogen cycling during post-agricultural succession and forest fertilization in the humid tropics. Ecosystems 4:67-84.

Espinosa-Carvajal, M; Contreras-Santos, J; Cadena, J; Martínez-Atencia, J; Jaramillo-Barrios, C; Hurtado, M. 2020. Flujos de metano en suelos con coberturas de pastos en el norte de Colombia. Agronomía Mesoamericana 31(2):291-309. DOI: 10.15517/ am.v31i2.38387.

FAO (Organización de las Naciones Unidas para la Alimentación y la Agricultura) y GTIS (Grupo Técnico Intergubernamental de Suelos). 2015. Estado Mundial del Re-curso Suelo (EMRS) - Resumen Técnico. Grupo Técnico Intergubernamental del Suelo y Organización de las Naciones Unidas para la Alimentación y la Agricultura. Organización de las Naciones Unidas para la Alimentación y la Agricultura y Grupo Técnico. s.p.

Gao, B; Ju, X; Su, F; Meng, Q; Oenema, O; Christie, P; Chen, X; Zhang, F. 2014. Nitrous oxide and methane emissions from optimized and alternative cereal cropping systems on the North China Plain: a twoyear field study. Sci. Total Environ. 472:112-124. DOI: 10.1016/j.scitotenv.2013.11.003.

González-Estrada, A; Camacho-Amador, M. 2017. Emisión de gases de efecto invernadero de la fertilización nitrogenada en México. Revista Mexicana de Ciencias Agrícolas 8(8):1733-1745. DOI: 10.29312/ remexca.v8i8.698.

Gulledge, J; Schimel, JP. 2000. Controls on soil carbon dioxide and methane fluxes in a variety of taiga forest stands in interior Alaska. Ecosystems 3:269-282.

Hendershot, W; Lalande, H; Duquette, M. 2007. Ion exchange and exchangeable cations 2 ed. In Carter, MR; Gregorich, EG (eds.). Soil Sampling and Methods of Analysis. Canada, Canadian Society of Soil Science. p. 197-207.

Herrero, M; Henderson, B; Havlík, P; Thornton, PK; Conant, RT Smith, P; Wirsenius, S; Hristov, AN; Gerber, P; Gill, M; Butterbach-Bahl, K; Valin, H; Garnett, T; Stehfest, E. 2016. Greenhouse gas mitigation potentials in the livestock sector. Nature Climate Change 6(5):452-461. DOI: 10.1038/nclimate2925.

Holdridge, LR. 2000. Ecología basada en zonas de vida. 5 ed. San José, Costa Rica, IICA. 225 p. 
IGAC (Instituto Geográfico Agustín Codazzi). 2006. Métodos analíticos del laboratorio de suelos. 6 ed. Bogotá, Colombia, Imprenta Nacional de Colombia. sp.

Lam, SK; Suter, H; Bai, M; Walker, C; Davies, R; Mosier, AR; Chen, D. 2018. Using urease and nitrification inhibitors to decrease ammonia and nitrous oxide emissions and improve productivity in a subtropical pasture. Science of The Total Environment, 644:1531-1535. DOI: 10.1016/j.scitotenv.2018.07.092.

LeBauer, DS; Treseder, KK. 2008. Nitrogen limitation of net primary productivity in terrestrial ecosystems is globally distributed. Ecology 89:371-379.

Liang, LL; Campbell, DI; Wall, AM; Schipper, LA. 2018. Nitrous oxide fluxes determined by continuous eddy covariance measurements from intensively grazed pastures: Temporal patterns and environmental controls. Agriculture, Ecosystems \& Environment 268:171-180. DOI: 10.1016/j.agee.2018.09.010.

Montenegro, J. 2020. Efecto de diferentes fuentes de nitrógeno en la emisión de óxido nitroso en plantaciones de café en Costa Rica. R. C. A. 54(2):111-130. DOI: 10.15359/rca.54-2.6.

Nakamura, S; Saliou, PS; Takahashi, M; Ando, Y; Subbarao, GV. 2020. La contribución de la rotación de raíces en la inhibición de la nitrificación biológica y su impacto en las arqueas oxidantes de amoníaco en cultivos de Brachiaria. Agronomía 10(7):1003. DOI: 10.3390/agronomy10071003.

Neff, JC; Bowman, WD; Holland, EA; Fisk, MC; Schmidt, SK. 1994. Fluxes of nitrous-oxide and methane from nitrogenamended soils in a Colorado alpine ecosystem. Biogeochemistry 27:23-33.

Nelson, D; Sommers, L. 1983. Total carbon, organic carbon and organic matter. 2 ed. In Page, A; Miller, R; Kenney, D (eds.). Methods of soil analysis. Part 2 Chemical and microbiological properties. Wisconsin, USA, American Society of Agronomy and Soil Science Society of America. p. 403-430.

Pastrana, I; Reza, S; Espinosa, M; Suárez, E; Díaz, E. 2011. Efecto de la fertilización nitrogenada en la dinámica del óxido nitroso y metano en Brachiaria humidicola (Rendle) Schweickerdt. Ciencia \& Tecnología
Agropecuaria 12(2):134-142. DOI: 10.21930/rcta. vol12_num2_art:223.

Pulido, A; Turriago, J; Jiménez, R; Torres, C; Rojas, A; Chaparro, N; López, A. 2016. Inventario nacional y departamental de gases efecto invernaderoColombia. Tercera Comunicación Nacional de Cambio Climático. IDEAM, PNUD, MADS, DNP, CANCILLERIA, y FMAM, Bogotá, Colombia. s.p.

Ravishankara, AR; Daniel, JS; Portmann, RW. 2009. Nitrous Oxide $\left(\mathrm{N}_{2} \mathrm{O}\right)$ : The Dominant Ozone-Depleting Substance Emitted in the 21 st Century. Science 326(5949):123-125. DOI: 10.1126/science.1176985.

Rivera, JE; Chará, J; Barahona, R. 2018. CH4, CO2 and $\mathrm{N} 2 \mathrm{O}$ emissions from grasslands and bovine excreta in two intensive tropical dairy production systems. Agrofor. Syst. 93(3):915-928. DOI: 10.1007/s10457018-0187-9.

Rondón, M. 2000. Land use and balances of greenhouse gases in Colombian Tropical Savannas. Ph.D. Thesis. NY, USA, Cornell University. 211 p.

Simon, PL; Dieckow, J; Zanatta, JA; Ramalho, B; Ribeiro, RH; van der Weerden, T; de Klein, CAM. 2020. Does Brachiaria humidicola and dicyandiamide reduce nitrous oxide and ammonia emissions from cattle urine patches in the subtropics?. Sci. Total Environ. 720:137-692. DOI: 10.1016/j.scitotenv.2020.137692.

Singh, N; Abagandura, GO; Kumar, S. 2020. Short-term grazing of cover crops and maize residue impacts on soil greenhouse gas fluxes in two Mollisols. Journal of Environmental Quality 49(3):628-639. DOI: $10.1002 /$ jeq2.20063.

Soil Survey Staff. 2014. Keys to soil taxonomy 12 ed. USDANatural Resources Conservation Service. USA, United States Department of Agriculture. 399 p.

Torres, CF. 2020. Fertilización nitrogenada, línea base y proyecciones de gases de efecto invernadero en Colombia". Colombia, CGIAR Research Program on Climate Change, Agriculture and Food Security (CCAFS). s.p.

Ussiri, D; Lal, R. 2012. Nitrous Oxide Sources and Mitigation Strategies. In Soil Emission of Nitrous Oxide and its Mitigation. Springer, Dordrecht. DOI: 10.1007/97894-007-5364-8_8.

Todos los derechos reservados. Universidad de Costa Rica. Este artículo se encuentra licenciado con Creative Commons Reconocimiento-NoComercial-SinObraDerivada 3.0 Costa Rica. Para mayor información escribir a rac.cia@ucr.ac.cr 
